\title{
Criminologie
}

\section{La détention provisoire au Québec : une pratique judiciaire courante}

\section{Marie-Luce Garceau}

Volume 23, numéro 1, 1990

Nouvelles violences à l'adolescence

URI : https://id.erudit.org/iderudit/017290ar

DOI : https://doi.org/10.7202/017290ar

Aller au sommaire du numéro

Éditeur(s)

Les Presses de l'Université de Montréal

ISSN

0316-0041 (imprimé)

1492-1367 (numérique)

Découvrir la revue

Citer cet article

Garceau, M.-L. (1990). La détention provisoire au Québec : une pratique judiciaire courante. Criminologie, 23(1), 117-134.

https://doi.org/10.7202/017290ar
Résumé de l'article

Little is known in Québec about detention before sentencing also known as provisionnel detention. This is a study of the population admitted to provisionnel detention between 1981 and 1985. The author presents the conceptual framework in which this study was undertaken and, with the help of statistical data, she draws a portrait of the population concerned. The author points out there is a disparity between the theory and practice of provisionnel detention. Furthermore, it is used disproportionately for marginal groups of detained persons. 


\title{
LA DÉTENTION PROVISOIRE AU QUÉBEC: UNE PRATIQUE JUDICIAIRE COURANTE ${ }^{1}$ \\ Marie-Luce Garceau ${ }^{2}$
}

\begin{abstract}
Little is known in Québec about detention before sentencing also known as provisionnel detention. This is a study of the population admitted to provisionnel detention between 1981 and 1985. The author presents the conceptual framework in which this study was undertaken and, with the help of statistical data, she draws a portrait of the population concerned. The author points out there is a disparity between the theory and practice of provisionnel detention. Furthermore, it is used disproportionately for marginal groups of detained persons.
\end{abstract}

\section{INTRODUCTION}

La détention provisoire est une mesure judiciaire peu et mal connue; elle est, à toutes fins pratiques, ignorée de toute l'administration de la justice criminelle au Québec. En effet, depuis une vingtaine d'années, l'attention de la justice, comme celle des chercheurs et analystes du système pénal, s'est surtout portée sur la nature et les visées différentielles des sentences, sur la peine de mort, la récidive, les jeunes contrevenants et les femmes criminalisées; une importante réalité a ainsi été négligée: celle des personnes détenues provisoirement dans les établissements de détention de juridiction québécoise.

Théoriquement, détenir provisoirement un individu avant qu'il ait été reconnu coupable se veut être une pratique résiduelle qui ne doit s'appliquer que lorsque cela est absolument nécessaire et indispensable ${ }^{3}$. Cependant, il semble que cela se pratique couramment au Québec. Nous inscrivant dans la perspective d'une utilisation plus sélective de l'incarcération en détention provisoire, nous croyons qu'il est nécessaire que celle-ci fasse l'objet d'un examen. À l'aide de données statistiques, il s'agira pour nous de mesurer

1. Les résultats présentés s'inscrivent dans le cadre d'une subvention de recherche du FCAR, volet «Équipe».

2. Chercheuse, Groupe de recherche et d'analyse sur les politiques et les pratiques pénales (GRAPPP), UQAM et professeure, École de service social de l'Université Laurentienne, Sudbury, Ontario, P3E 2C6. Le présent article est tiré du mémoire de maîtrise de l'auteure. Je tiens à remercier Shirley Roy, chercheuse, GRAPPP, et Danielle Laberge, professeure, UQAM, pour leur précieuse collaboration.

3. Comité canadien de la réforme pénale et correctionnelle, 1969; Commission de réforme du droit du Canada, 1988. 
l'ampleur de la détention provisoire. Nous soulignerons le caractère usuel du recours à cette mesure et insisterons sur la précarité financière des personnes détenues provisoirement. Pour ce faire, nous présenterons, en premier lieu, une définition de la détention provisoire. Nous verrons, par la suite, cette mesure dans son contexte théorico-légal en mettant un accent particulier sur les motifs utilisés afin de détenir provisoirement un accusé. Nous mettrons en perspective les différentes analyses réalisées jusqu'à maintenant sur cette disposition judiciaire. Nous aborderons ensuite certains aspects déterminants qui précèdent le renvoi d'une personne accusée au tribunal, soit l'arrestation et l'acte d'accusation ainsi que l'atmosphère de culpabilité qui règne tout au long de la procédure pénale. Finalement, nous nous pencherons sur l'évolution de la détention provisoire ainsi que sur quelques caractéristiques sociales, démographiques et judiciaires de la population qu'elle touche.

\section{LA DÉTENTION PROVISOIRE: UNE DÉFINITION}

Dans le langage commun, comme dans les études présentées par les chercheurs et analystes du système pénal, on utilise souvent et sans distinction, des termes tels que la mise sous garde, la détention préventive, la détention avant procès ou encore la détention provisoire. Toutefois, il est erroné d'employer ces termes indistinctement puisque chacun correspond à une pratique particulière qu'il importe de caractériser.

La détention provisoire diffère de la détention policière, ou de la «mise sous garde», ou de la «détention sous garde» ${ }^{4}$. Cette dernière ne s'applique qu'à la période de détention allant de l'arrestation d'un présumé infracteur à sa comparution initiale devant un juge de paix ${ }^{5}$. La détention provisoire fait ainsi suite à la mise sous garde. Elle se distingue aussi de ce que l'on appelle communément la détention préventive en ce sens qu'elle n'est pas considérée, juridiquement, comme une peine. Théoriquement, la mesure de détention préventive ne peut être appliquée que suite à une condamnation, dans le cas de délinquance dangereuse et les modalités de libération en sont précises ${ }^{6}$. Le terme détention provisoire peut aussi servir à désigner la pratique de «détention avant procès »? Toutefois, ce terme ne suffit pas à montrer toute la réalité

4. Saint-Onge-Poitevin, L., (1988), Code criminel, articles 452,453 et 453.1 . Veuillez noter que nous avons utilisé la version 1988.

5. Le délai normal entre la période d'arrestation et la comparution étant de 24 heures, cette période peut être allongée si le juge de paix n'est pas disponible. Code criminel, article 454 (1(a) et b).

6. Code criminel, partie XXI, article 687 .

7. Le Code criminel, article $457(7)$, désigne la détention provisoire sous le terme de «détention d'un prévenu sous garde». 
de cette mesure judiciaire. Il ne s'agit pas ici d'une forme de détention avant un procès mais plutôt d'une période d'incarcération provisoire qui peut être plus ou moins longue, couvrant d'une partie à toute la durée d'un procès, selon les décisions émises par le tribunal.

Suite à ces brèves explications terminologiques, nous retenons que l'utilisation du terme détention provisoire semble de mise. Nous entendrons par cela la période de détention résultant d'un mandat judiciaire, émis par le juge d'un tribunal, avant le prononcé d'un jugement définitif sur la culpabilité et l'imposition d'une sentence et, par détenu provisoire, la personne à qui s'applique cette mesure.

\section{LES MODALITÉS D'APPLICATION DE LA DÉTENTION PROVISOIRE}

Lors de la comparution d'un prévenu devant le tribunal, si celui-ci n'enregistre pas un plaidoyer de culpabilité, le juge procède habituellement à l'audition de l'enquête sur la mise en liberté provisoire, communément appelée enquête sur cautionnement. La couronne doit alors prendre position et présenter, dans le cas d'une objection, les motifs justifiant la détention provisoire de l'accusé. Ainsi, sous réserve des exceptions au régime normal ${ }^{8}$, le juge de paix doit ordonner soit la libération, soit la détention provisoire du prévenu. Il n'existe que deux motifs pouvant justifier une décision de non-libération:

a) la détention du prévenu est nécessaire pour assurer sa présence au tribunal; c'est le motif principal; et,

b) si la détention n'est pas justifiée pour le motif principal, la détention du prévenu est nécessaire dans l'intérêt public ou pour la protection de celui-ci, compte tenu de toutes les circonstances, y compris la probabilité marquée que le prévenu, s'il est mis en liberté, commette une infraction criminelle ou nuise à l'administration de la justice ${ }^{y}$; c'est le motif secondaire.

Si le juge estime nécessaire l'incarcération en détention provisoire pour le motif principal, le prévenu sera alors interrogé sur un ensemble de critères dont, notamment, l'existence d'une adresse fixe, la plus ou moins grande stabilité de résidence et d'emploi, la présence d'attaches affectives provenant de la famille (parents, conjoint-e, enfants, amis et proches) et autres (Grenier, Tarasofsky, et al., 1987). On peut constater que les éléments pris en compte dans la décision d'imposer la détention provisoire, peuvent avoir pour effet

8. Nous faisons référence aux situations où un plaidoyer de culpabilité est enregistré et accepté par un juge de paix, aux exceptions concernant les situations pour lesquelles le juge de paix doit ordonner la détention provisoire (Code criminel, articles 457 et 457 (5.1)) et à certaines infractions en vertu de la Loi sur les stupéfiants.

9. Code criminel, article $457(7)$. 
de sélectionner une partie de la clientèle qui sera détenue provisoirement dont, tout particulièrement, les individus aux prises avec des problèmes d'ordre socio-économique, notamment, les sans-emploi, les chômeurs, les itinérants ou encore les personnes affichant des problèmes de santé mentale, etc. Ainsi, les individus qui n'ont ni résidence, ni emploi, qui ne peuvent répondre aux critères du motif principal, risquent fort d'être détenus provisoirement.

Par contre, si le juge de paix estime nécessaire l'incarcération d'un prévenu pour le motif secondaire, il prendra en considération toutes les circonstances entourant à la fois l'infracteur et l'infraction. Les critères alors examinés sont, entre autres, la menace que l'accusé représente pour les témoins, ses antécédents judiciaires, le type d'infraction commise et les circonstances de la perpétration, sa personnalité dont sa tendance au comportement violent, etc. (Grenier, Tarasofsky et al., 1987:55). L'examen de ces divers éléments doit permettre au juge de déterminer si la mise en liberté de l'accusé peut s'effectuer sans danger et si le tribunal peut être assuré de la présence du prévenu à toutes les étapes de la procédure judiciaire.

L'interprétation par le système juridique de la notion de danger a soulevé bon nombre de critiques. Plusieurs auteurs remettent largement en question la définition même de ce qui est dangereux (Montandon, 1979). D'autres critiquent la faible validité et la fidélité douteuse des prévisions tant cliniques que statistiques de la dangerosité (Debuyst, 1977; Dozois, Lalonde et Poupart, 1981). Certains, plus sévères, mettent en lumière l'impuissance à reconnaître les caractéristiques de la dangerosité et l'impossibilité de prédire un comportement délictueux, sans identifier à tort, comme dangereux, un grand nombre d'individus non violents (Marceau, 1986). Cependant, quoi qu'en disent les critiques, il semble que la dangerosité occupe toujours une place importante et que le système pénal ne peut se passer de cette notion puisqu'elle est un des instruments essentiels de la gestion de la délinquance.

\section{LA DÉTENTION PROVISOIRE ET L'ISSUE DE LA PROCÉDURE PÉNALE}

Au Canada, nous devons à Friedland (1965) la première et la plus importante étude sur la détention provisoire; se sont ensuite prononcés Koza et Doob (1975a, 1975b) puis Doob et Cavoukian (1977) et quelques autres. Les études menées aux États-Unis ne diffèrent pas des analyses canadiennes et les conclusions qu'elles présentent sont similaires ${ }^{10}$. Ainsi, depuis Morse et Beattie (1932), on souligne l'importance du nombre de personnes détenues provisoirement et la nécessité d'une utilisation restreinte de cette mesure, au profit d'un renforcement du système de libération à toutes les étapes décisionnelles

10. Goldkamp, dans son ouvrage Two Classes of Accuses (1979), présente un bilan de la littérature américaine, plus particulièrement en matière de cautionnement. 
du processus pénal. Par ailleurs, l'interprétation de la relation entre la détention provisoire et l'issue de la procédure pénale est au centre de toutes ces analyses. L'ensemble des auteurs s'entendent pour montrer qu'il existe un traitement discriminatoire à l'égard des personnes détenues provisoirement quant aux plaidoyers de culpabilité, aux déclarations de culpabilité ou aux sentences prononcées.

Bien que l'on s'entende sur ce lien, les auteurs se distinguent sur le plan des variables explicatives ainsi que sur la force des relations existantes; trois modèles explicatifs sont offerts.

Le premier suppose que la détention provisoire a une influence directe et négative sur la déclaration de culpabilité que prononce un individu ainsi que sur le prononcé de sa sentence (Friedland, 1965; Koza et Doob, 1975a; Mackaay, 1976; Doob et Cavoukian, 1977; Fischer, 1986). La pression que constitue la période de détention provisoire porte certains détenus à enregistrer un plaidoyer de culpabilité même si ce n'était pas là l'intention initiale. Cette pression, on ne la retrouve pas dans le cas de personnes qui se présentent devant le tribunal en état de liberté. Les auteurs reconnaissent le nombre élevé de plaidoyers de culpabilité dont les proportions varient entre $70 \%$ et $90 \%$ de l'ensemble des causes entendues par les tribunaux. La détention provisoire n'est certes pas la seule raison qui entraîne un individu à enregistrer un plaidoyer de culpabilité, elle demeure pourtant un des critères les plus importants". On constate, de plus, que le fait d'être en détention provisoire constitue un événement qui crée une atmosphère psychologique négative qui opère au détriment de l'accusé. Concrètement, le fait d'être détenu devient un indicateur de culpabilité qui a des implications réelles dans les décisions du tribunal: il prédispose défavorablement le juge et influence le type de sentence prononcée par celui-ci.

Le second modèle met l'accent sur la gravité de l'infraction commise, le type d'accusation portée et les antécédents judiciaires de l'accusé (Koza et Doob, 1975a; Leigh, 1986; MacKaay, 1976). Ces facteurs, déterminants dans la décision de détenir provisoirement un accusé, s'additionnant à la période de détention, augmentent les probabilités de la déclaration de culpabilité et accroissent l'importance de la sentence. Les tenants de ce point de vue soutiennent, en outre, que la détention provisoire place, directement ou indirectement, la personne dans une situation désavantageuse et affecte ses capacités d'accès à des informations pertinentes entravant ainsi la préparation et la présentation d'une défense adéquate (MacKaay, 1976).

11. D'autres facteurs doivent être pris en considération tels que le désir de l'individu de plaider coupable, le fait que l'avocat soit un spécialiste de cette forme de négociation, le nombre de causes dont l'avocat de la défense doit s'occuper, etc. Poirier, 1987:65.a. 
Les tenants du troisième modèle soutiennent que la détention provisoire perd toute son influence quand on fait intervenir six variables contrôles. Ces variables sont peu déterminantes quand elles sont prises isolément, mais ensemble, elles affectent avec plus ou moins de sévérité les décisions du tribunal. Ces variables sont: la gravité de l'infraction, l'existence d'un ou de plusieurs mandats d'arrestation, le nombre d'arrestations antérieures, le fait qu'un accusé soit en libération conditionnelle ou en probation et le nombre d'infractions initialement portées contre l'accusé (Goldkamp, 1979). Toutefois, malgré le fait que l'on mette l'accent sur des variables contrôles, on ne fait pas la preuve de l'inexistence d'un lien entre le fait d'être détenu provisoirement et l'issue négative de la procédure pénale. Au contraire:

[...] the relationship in question may or may not exist and may or may not be shown to be spurious, depending on the kind of decision and the place of that decision in the criminal process. The verdict, then, must be «it depends». (Goldkamp, 1979:103-104)

Malgré la variété des énoncés, on peut affirmer que l'ensemble des écrits tend à démontrer l'influence de la détention provisoire sur le plaidoyer de culpabilité, la déclaration de culpabilité ou la sentence à l'égard des personnes détenues provisoirement.

Cela dit, la majorité des auteurs qui ont abordé la détention provisoire se sont limités à l'examen du déroulement de cette procédure. Il nous semble important, afin de mieux comprendre cette mesure, de mettre en lumière deux mécanismes déterminants qui précèdent le renvoi d'une cause au tribunal, soit l'arrestation et l'acte d'accusation. Par ailleurs, plusieurs recherches ont analysé l'importance de l'atmosphère de culpabilité qui règne autour du prévenu pendant le déroulement de la procédure pénale. Ces divers aspects semblent, comme nous le verrons, influer sur les décisions prises à l'égard des détenus provisoires.

\section{DE L'ARRESTATION À LA DÉTENTION PROVISOIRE: UNE COURSE À OBSTACLES}

\section{L'ARRESTATION}

Le processus judiciaire commence bien avant que la personne ne se présente devant le tribunal et certains auteurs ont soulevé l'importance et l'influence de l'arrestation et de la mise sous garde d'un prévenu sur le déroulement de la procédure pénale. Selon Morden (1980), les facteurs pris en considération par les agents de la paix, lors de l'arrestation, jouent un rôle important quant aux décisions de procéder à celle-ci ainsi qu'à la mise sous 
garde d'un suspect. Dans cette recherche torontoise, Morden expose de façon explicite les critères associés à la décision policière en matière de cautionnement. Ces critères sont: les condamnations antérieures du prévenu, son expérience antérieure de détention, sa situation d'emploi, sa conduite envers les policiers, la gravité de l'infraction, le type de victime en cause, l'existence d'un mandat d'arrestation et la prise d'une déclaration par les policiers.

Ces mêmes critères permettent une première différenciation dans la population qui sera amenée à se présenter devant le tribunal au moment de l'enquête sur cautionnement : une première sélection entre les personnes en liberté et celles mises sous garde lors de l'arrestation. Levy (1985) note, relativement à ces dernières, l'influence de l'arrestation et de la mise sous garde sur la continuation de la procédure pénale:

[...] le processus pénal est très largement dominé par ce que l'on pourrait appeler la politique et les pratiques policières qui, par les choix qu'elles impliquent, circonscrivent étroitement les décisions ultérieures et ne laíssent aux tribunaux (dans le cas du Québec) qu'un rôle relativement mineur dans la production de la structure sociale spécifique de la population finalement jugée. (p.65)

Hétu (1976) souligne, par ailleurs, que les policiers effectuent, au moment de l'arrestation, une sélection différente selon qu'il s'agit des quartiers huppés ou des zones grises de la ville. Le fait que les «pauvres» soient surreprésentés dans le système pénal serait dû, en partie, à l'intervention initiale des policiers.

En plus d'être importantes dans toute judiciarisation d'une affaire, les décisions policières influent, selon Grosman (1969), sur les recommandations de la couronne lors de l'enquête sur cautionnement. Grosman mentionne à ce sujet que :

The prosecutor's recommendation itself is not the product of independant information relating to the roots of the accused in the community. Because of the need for a speedy decision it is often based on the advice of the police officer in charge of the case, or the court liaison officer. (Grosman, 1969:53)

Les facteurs qui influencent la recommandation de la couronne lors de l'enquête sur cautionnement sont, par ordre d'importance, la recommandation de la police dans une affaire, les antécédents criminels de l'accusé, la gravité de l'offense, la possibilité que l'infracteur commette une autre offense s'il est libéré, la possibilité qu'il influence les témoins, ses racines dans la communauté et son apparence physique. Bref, il semble que la décision de la couronne soit plutôt «d'ordre technique, cependant qu'il revient à la police d'opé- 
rer une sélection, sur une base sociale, de la population prise en charge». (Levy, $1985: 71$ )

\section{LA DÉNONCIATION ET LE DÉPÔT DE L'ACTE D'ACCUSATION: LE RÔLE DU POLICIER}

L'acte d'accusation, tant dans sa rédaction que dans son dépôt, joue un rôle important dans les décisions à prendre au sujet des prévenus (Ericson, 1981a, 1981b, 1982; Ericson et Baranek, 1982, Leighton et Del Buono, 1986; Falardeau, Tarasofsky et al., 1987). Certains auteurs remarquent la faiblesse générale de ces dénonciations. Or, c'est à partir de ce document que le procureur de la couronne doit formuler une ou des dénonciations contre un prévenu. Comme ce rapport émane d'une personne qui n'a pas été nécessairement témoin de l'incident (le policier), il ne contient, généralement, que les éléments de preuve inculpatoire à l'égard du suspect. On a aussi remarqué que les policiers ont tendance à terminer une enquête quand ils estiment qu'une infraction est résolue, sans pour autant évaluer la légalité des preuves accumulées au cours de cette enquête (Falardeau, Tarasofsky et al., 1987). Leighton et Del Buono (1986) mentionnent que certaines études ont permis de «confirmer et d'expliquer la pratique courante des agents de police de porter plus d'accusations que la situation ne l'exige» (p.437). Par voie de consêquence, un prévenu peut être incarcéré en détention provisoire à cause de la nature même de ce rapport et des accusations qui y sont portées.

\section{L'ATMOSPHÈRE DE CULPABILITÉ AUTOUR DU PRÉVENU}

Dans la Charte canadienne des droits et libertés, on peut lire que:

Tout inculpé a le droit (...) d'être présumé innocent tant qu'il n'est pas déclaré coupable, conformément à la loi, par un tribunal indépendant et impartial à l'issue d'un procès public et équitable. (article 11d)

Dans les faits, un tel portrait ne correspond pas à la situation qui prévaut. Certaines êtudes ont démontré que, lorsqu'ils effcctuent une arrestation, les policiers sont généralement convaincus de la culpabilité de l'individu qu'ils amènent (Grosman, 1969). C'est donc, en premier lieu, lors de l'arrestation que s'établit la présomption de culpabilité envers le prévenu. En outre, les policiers tendent à faire accepter leur point de vue et à influencer fortement les décisions de la couronne. Il semble bien que les procureurs partagent cette conviction bien que la preuve n'en ait pas encore été faite (Grosman, 1969). D'autre part, Robert (1984) mentionne qu'il y aurait lieu de penser que les liens étroits qui s'établissent entre la police et la couronne puissent aussi se répercuter à d'autres niveaux, à savoir entre la couronne et les juges. Cette hypothèse se fonde sur le fait que «la justice tend à incarcérer immédiatement 
là ou la police a arrêté et détenu» (Robert, 1984:19). Il y aurait donc un possible enchaînement entre la présomption de culpabilité, les décisions policières et celles des procureurs et des juges et le recours à l'utilisation de moyens cœrcitifs tels que la mise sous garde et l'application de la détention provisoire.

À la lumière de cette brève recension des écrits, nous pouvons constater que jusqu'à maintenant les études entreprises par les chercheurs ont mis en relief le déroulement procédural de la détention provisoire et ses conséquences pour les prévenus incarcérés. Toutefois, la grande majorité d'entre elles ont porté sur des facteurs judiciaires et institutionnels. Il nous semble que c'est fausser toute analyse sur la détention provisoire que de ne considérer que ces aspects. Il est, selon nous, tout aussi important de regarder des facteurs tant humains qu'organisationnels. D'une part, certains facteurs propres au système judiciaire tels que le pouvoir discrétionnaire des différents professionnels du système judiciaire, les buts du système, les relations informelles qui se développent dans l'environnement du tribunal et qui contrôlent le processus décisionnel, le déroulement expéditif des événements au tribunal et, d'autre part, certaines caractéristiques propres à l'accusé telles que son apparence, le manque de garanties de sa représentation ou son appartenance de classe, voire le phénomène de pauvreté, sont autant d'éléments qui influencent, selon toute probabilité, non seulement le fonctionnement du tribunal, mais aussi les décisions à l'égard d'un accusé détenu provisoirement.

Poussant plus avant notre réflexion, nous soulevons l'hypothèse de l'existence d'une gestion discriminatoire du système pénal, par le biais de la détention provisoire, à l'endroit des personnes socio-économiquement défavorisées. Ce n'est là une réalité ni nouvelle, ni originale: nos établissements de détention regorgent de personnes présentant cette caractéristique. Certaines études ont démontré cette réalité. Le système pénal est discriminatoire envers les économiquement faibles (Campeau, 1976), et la répression de la criminalité est intimement liée au phénomène de la pauvreté (Campeau, 1976; Laberge et Théorêt, 1986). Il nous semble possible de penser que dans l'exercice de la mesure de détention provisoire, on retrouverait ce même phénomène.

Par ailleurs, nous savons que les prisons québécoises accueillent des individus ayant surtout commis des délits mineurs (Hattem, 1980; Cousineau, Laberge et Théorêt, 1986; Néron, 1988) et un doute profond s'installe lorsque l'on constate le refus de mettre en liberté provisoire certaines catégories d'infracteurs. Comme le mentionnent Laberge et Théorêt (1986):

[...] les représentations habituellement véhiculées et selon lesquelles les personnes que l'on retrouve dans les établissements carcéraux du pays sont des individus dangereux ayant commis des crimes graves et 
présentant une menace certaine pour la société, perdent, pour une bonne part, leur crédibilité. (p.37)

Finalement, cette étude de la détention provisoire nous amène à suggérer que, contrairement à l'image répandue qui veut qu'elle ne s'applique que lorsqu'elle est absolument indispensable, la détention provisoire serait une pratique relativement courante.

\section{UN APERÇU DE LA SITUATION}

Afin de répondre à notre objectif concernant l'ampleur de la détention provisoire au Québec, il nous fallait recenser l'ensemble de la clientèle y étant soumise. La première source de données que nous utilisons répond partiellement à ces impératifs. Il s'agit des données diffusées par les Services correctionnels du Québec pour les dix dernières années (1979-80 à 1988-89). Ces informations étant limitées, nous avons dû retenir une seconde source d'information afin de procéder à l'analyse des caractéristiques sociodémographiques, institutionnelles et judiciaires de la population visée par cette mesure. Il s'agit d'une bande informatisée qui nous est foumie par le Groupe de recherche et d'analyse sur les politiques et les pratiques pénales (GRAPPP) pour les années allant de 1981 à 1985 et qui contient des données quantitatives concernant l'ensemble des individus ayant été admis dans les établissements de détention de juridiction québécoise.

\section{LA DÉTENTION PROVISOIRE SELON LES SERVICES CORRECTIONNELS AU QUÉBEC ${ }^{\prime 2}$}

L'examen des données statistiques des Services correctionnels au Québec, concernant l'ensemble des admissions au cours des dix dernières années (1979-80 à 1988-89), nous indique que 373338 admissions ont été enregistrées au cours de cette période. Les détenus provisoires représentent 174921 de ces admissions; les 198417 restantes sont celles de personnes admises en détention punitive. C'est donc dire qu'au cours des dix dernières années, près de $47 \%$ des admissions dans les prisons québécoises ont été le fait de personnes détenues provisoirement ${ }^{13}$.

12. Afin de situer le phénomène de la détention provisoire par rapport à l'ensemble des pays occidentaux, voir: Doyon, 1989, pp.25-34; Déviance et Société, 1986. Par ailleurs, afin de refléter la réalité de l'application de la mesure de détention provisoire, pour le Québec, nous avons exclu la population des personnes condamnées et admises dans les établissements de détention de juridiction fédérale.

13. Il faut comprendre qu'il s'agit du volume d'admissions enregistrées dans les prisons québécoises et non du nombre réel d'individus ayant été admis puisqu'un seul individu peut avoir été détenu provisoirement à plusieurs reprises au cours de cette période. Le volume des admissions est ici capital puisqu'il reflète mieux, selon nous, la réalité de l'utilisation de la mesure. Afin de connaître le compte réel de contrevenants admis dans les prisons québécoises, voir: Statistique Canada, 1987. 
La distribution annuelle de l'ensemble de ces admissions (Tableau 1) permet de constater une augmentation de l'ordre de $85 \%$ des admissions en détention provisoire entre les années juridiques 1979-80 et 1988-89. Cet accroissement s'effectue graduellement tout au long des dix années. Toutefois, il est plus marqué à la fin de la période et atteint, pour l'année 1988-89, près de $59 \%$ du total de l'ensemble des admissions dans les prisons québécoises. Alors que le volume des admissions en détention provisoire augmente radicalement, la détention punitive diminue légèrement ( $3 \%$ ) entre 1979-80 et 1988-89. Ainsi, comparativement aux admissions en détention punitive, celles des détenus provisoires occupent une place de plus en plus importante dans notre système carcéral.

\section{TABLEAU 1}

Distribution annuelle des admissions provisoires et punitives dans l'ensemble des établissements de détention de juridiction provinciale (Québec, 1978-79 à 1988-89)

\begin{tabular}{lrrrrr}
\hline Année & $\begin{array}{c}\text { Détention provisoire } \\
\text { (nombre) }\end{array}$ & $(\%)$ & $\begin{array}{c}\text { Détention punitive } \\
(\text { nombre })\end{array}$ & Total \\
\hline $1988-89$ & 25680 & 58,9 & 17890 & 41,6 & 43570 \\
$1987-88$ & 19278 & 54,8 & 15927 & 45,2 & 35205 \\
$1986-87$ & 17939 & 51,6 & 16825 & 48,4 & 34764 \\
$1985-86$ & 17419 & 48,6 & 18437 & 51,4 & 35856 \\
$1984-85$ & 15763 & 47,7 & 17267 & 52,3 & 33030 \\
$1983-84$ & 16926 & 40,5 & 24888 & 59,5 & 41814 \\
$1982-83$ & 16511 & 38,2 & 26741 & 61,8 & 43252 \\
$1981-82$ & 16090 & 42,7 & 21620 & 57,3 & 37710 \\
$1980-81$ & 15437 & 43,1 & 20388 & 56,9 & 35825 \\
$1979-80$ & 13878 & 42,9 & 18434 & 57,1 & 32312 \\
Total & 174921 & 46,9 & 198417 & 53,1 & 373338 \\
\hline
\end{tabular}

Source: Services cortectionnels, 1979-80 à 1988-89.

Comme nous l'avons mentionné auparavant, la détention provisoire se veut, en principe, une mesure résiduelle. Toutefois, les données recueillies nous confirment qu'elle est utilisée fort couramment et que son usage s'accroît au cours des dix dernières années.

\section{CERTAINES CARACTÉRISTIQUES DES DÉTENUS PROVISOIRES ENTRE 1981 ET 1985}

Les données offertes par les Services correctionnels au Québec ne nous permettaient pas de présenter certaines caractéristiques concernant les personnes détenues provisoirement. Pour ce faire, nous utiliserons celles que 
nous offre le GRAPPP. Avant de procéder à cet examen, il importe de souligner que ces données statistiques ont été produites par l'extraction des informations contenues sur des bandes informatiques fournies par le Service de l'informatique du ministère de la Justice du Québec. Suite à une série d'opérations, nous n'avons retenu que les informations traitant de la population détenue provisoirement pour la période allant de 1981 à $1985^{14}$.

Selon cette seconde source de données, les admissions en détention provisoire et punitive au Québec, de 1981 à 1985, s'élèvent à 173746 (Tableau 2). De ce nombre, $46,5 \%$ sont des admissions en détention provisoire et $53,5 \%$ en détention punitive pour les mêmes cinq années. Toutefois, alors que l'on remarque une baisse dans le volume des admissions punitives de $17,8 \%$ entre 1981 et 1985 , les admissions provisoires affichent une hausse de $4,7 \%$.

TABLEAU 2

Distribution annuelle des admissions en détention provisoire et punitive. GRAPP, 1981-1985.

\begin{tabular}{lrrrrrr}
\hline Année & 1981 & 1982 & 1983 & 1984 & 1985 & Total \\
\hline Détenus provisoires & 15413 & 16091 & 17351 & 15715 & 16139 & 80709 \\
& 19,1 & 19,9 & 21,5 & 19,5 & 20,0 & 100,0 \\
& 46,2 & 41,7 & 43,8 & 50,2 & 52,3 & 46,5 \\
Détention punitive & 17913 & 22529 & 22308 & 15588 & 14719 & 93037 \\
& 19,3 & 24,2 & 24,0 & 16,7 & 15,8 & 100,0 \\
& 53,8 & 58,3 & 56,2 & 49,8 & 47,7 & 53,5 \\
Total & 33326 & 38620 & 39659 & 31283 & 30858 & 173,746 \\
& 19,2 & 22,2 & 22,8 & 18,0 & 17,8 & 100,0 \\
Valeurs présentes: $100 \%$ & & & & & & \\
\hline
\end{tabular}

Source: Garceau, 1988: 149

L'examen des caractéristiques sociodémographiques des personnes détenues provisoirement de 1981 à 1985 montre que la très grande majorité sont des hommes $(93,8 \%)$, célibataires $(79,4 \%)$, jeunes (l'âge moyen étant de 27,6 ans), résidant au Québec $(69,1 \%)$, dont la profession se trouve quasi

14. Les procédures et remarques méthodologiques concernant cette bande informatisée sont traitées dans Cousineau, Laberge et Thêorêt, 1986:15-43 et Garceau, 1988:108-116. 
essentiellement dans les catégories pouvant être considérées comme «manuelles» $(55,1 \%)^{15}$.

Par ailleurs, seuls $27,8 \%$ des prévenus ont mentionné occuper un emploi au moment de leur admission dans les établissements de détention québécois, pendant la période de 1981 à 1985 . Il va sans dire que nous ne pouvons savoir ce qu'il advient des $72,2 \%$ des admissions restantes et que nous ne pouvons clairement nous prononcer puisqu'il semble que seules les réponses affirmatives aient été enregistrées. Toutefois, ce pourcentage pourrait peut-être constituer une approximation du nombre d'individus qui peuvent, selon toute probabilité, rejoindre le groupe des sans-emploi, des chômeurs, des prestataires de l'aide sociale et autres. Afin d'appuyer cette tendance, nous avons consulté une analyse concernant la population détenue provisoirement au Centre de prévention de Montréal (616 sujets) et effectuée pendant la période du 15 avril 1986 au 16 juillet de la même année (Doyon, 1989). Cette étude signale que seuls $37,0 \%$ des prévenus disent retirer un revenu lié à un emploi au moment de leur admission dans ce centre. Les $63,0 \%$ restants se partagent entre les prestataires de l'aide sociale $(38,6 \%)$, ceux qui mentionnent n'avoir aucune source de revenus ou une source de revenus considérée illégale $(12,7 \%)$, les bénéficiaires de prestations de l'aide sociale et de la Commission de la sécurité et de la santé au travail $(9,8 \%)$ et, finalement, les rentiers, étudiants et autres $(2,0 \%)$. (Doyon, 1989:84)

Si nous rappelons le fait que l'intervention initiale des policiers dans une affaire peut permettre la surreprésentation dans les prisons de personnes dont les moyens économiques sont faibles, voire inexistants, et que les décisions policières influent sur les recommandations de la couronne lors de l'enquête sur cautionnement, nous pouvons probablement comprendre, partiellement, comment cette mesure peut viser certaines catégories de justiciables dont la caractéristique principale est leur appartenance aux couches socioéconomiquement défavorisées de la société.

Nous avons constaté, au cours des années 1981 à 1985, une diminution dans l'ouverture de nouveaux dossiers carcéraux (première admission dans une institution carcérale) au profit d'une augmentation du nombre des admissions attribuées aux individus qui ont déjà été admis dans le système carcéral par le passé. Alors que les individus admis une première fois comptent pour $39,6 \%$ en 1981 , ils représentent $35,9 \%$ en 1985 . Un renversement de cette perspective indique que $60,4 \%$ des admissions en détention provisoire sont, pour l'année 1981, celles de personnes qui n'en sont pas à leur premier séjour

15. Les pourcentages les plus importants dans cette catégorie vont, par ordre décroissant, aux travailleurs de métier, d'artisanat, de production ouvrière, aux mancuvres, aux ouvriers de la construction et aux travailleurs miniers. Garceau, 1988:162-164. 
dans une institution carcérale; en 1985 , ce pourcentage grimpe à $64,1 \%$ des admissions.

Le retour dans le système carcéral, à titre de détenu provisoire, peut s'expliquer par l'importance des antécédents judiciaires dans la décision de détenir provisoirement un accusé. Plusieurs recherches ont déjà souligné, et le Code criminel le stipule clairement, que le casier judiciaire est un critère important, parmi tant d'autres, dans l'examen de la décision de libération ou d'incarcération d'un accusé. Ces réadmissions signalent un problème majeur dont il faut chercher les causes. Par ailleurs, les individus qui ont déjà séjourné dans une institution carcérale, quelle que soit la durée de ce séjour, sont, selon toute probabilité, plus susceptibles d'être pris en charge par ces mêmes institutions par le biais de la détention provisoire.

Quant à l'étude des infractions dont sont accusés les prévenus au moment de leur admission, nous remarquons que ce sont les infractions au Code criminel qui entraînent la majeure partie des ordonnances de détention provisoire. Celles-ci atteignent $84,9 \%$ pendant la période à l'étude. Ces délits sont suivis de loin par les infractions aux Statuts fédéraux $(9,1 \%)$, aux Règlements municipaux $(4,7 \%)$ et, finalement, aux Lois du Québec $(0,7 \%)$. Au cours des années 1981 à 1985, nous constatons une diminution du nombre des admissions en détention provisoire pour des infractions en vertu des Règlements municipaux $(3,4 \%)$ au profit d'un accroissement des infractions relevant du Code criminel $(4,2 \%)$, les deux autres juridictions de délits demeurant relativement stables.

La répartition en termes de catégories de délits dont sont accusés les détenus provisoires révèle que, par ordre d'importance, pour la période entre 1981 et 1985 , les délits contre les biens, sans violence, entraînent $47,1 \%$ des admissions en détention provisoire au Québec. Au second rang, nous retrouvons les infractions «autres ${ }^{16}$ » au Code criminel $(19,1 \%)$, puis les diverses infractions «autres ${ }^{17}$ contre la personne $(12,9 \%)$, les infractions relatives aux drogues $(8,6 \%$ ), les infractions à la circulation routière $(6,1 \%)$, les infractions aux règlements municipaux $(3,4 \%)$, les infractions contre la personne $(2,0 \%)$ et finalement, les infractions de nature administrative $(0,8 \%)$.

Nonobstant une quelconque apparence de gravité des délits lorsque nous référons à la juridiction ou aux catégories des délits, la mise en détention provisoire, liée à certaines de ces catégories, nous paraît discutable. Nous pou-

16. Ces infractions au Code criminel comprennent les catégories suivantes: a) mœurs, paris et prostitution, b) armes à feu, c) absence illégale et/ou désobéissance à la Cour, d) complot, complicité, e) inconduite et f) autres.

17. Les autres crimes contre la personne se présentent dans les catégories suivantes: a) autres offenses sexuelles, b) blesser, c) voies de fait, d) vol qualifié. 
vons certainement nous interroger sur la nécessité de l'utilisation de cette mesure pour une partie des infractions sans violence contre les biens, une bonne part des infractions à la circulation routière, des infractions aux règlements municipaux et des infractions de nature administrative. Comme l'ont déjà mentionné Cousineau, Laberge et Théorêt (1986), certaines de ces infractions :

[...] ne nous apparaissent pas a priori viser des personnes qui présenteraient un risque important pour la société ou qui tenteraient de se soustraire aux procédures judiciaires entreprises à leur encontre. (p. 174)

\section{CONCLUSION}

Cet examen de la mesure judiciaire de la détention provisoire au Québec brosse un bref tableau de la situation. Ce portrait nous permet d'entrevoir qu'entre le bien-fondé de cette mesure issue de la bonne volonté des législateurs et son application quotidienne par les divers agents du système judiciaire, entre la théorie et la pratique, existe une distance profonde.

Cette disparité, nous la retrouvons dans le principe qui exige que la détention provisoire ne soit utilisée qu'en dernier recours. Les données présentées nous font douter d'une utilisation exceptionnelle et modérée de cette mesure de coercition; au contraire, elles semblent en souligner le caractère plutôt usuel. Comme l'a signalé le Comité canadien de la réforme pénale et correctionnelle en 1969, la détention injustifiée est une atteinte aux droits de la personne. Certains auteurs ont souligné les conséquences néfastes, tant personnelles que sociales, qu'entraîne une période d'incarcération (Landreville, Blankevoort et Pires, 1981). Ces conséquences s'appliquent, selon nous, aux détenus provisoires. Nous pensons, entre autres, aux problèmes qu'occasionne l'immersion d'un prévenu dans un établissement de détention, aux bouleversements des relations familiales, aux ruptures sociales, à la perte de biens, à la perte probable d'un emploi, à de possibles conséquences psychologiques, à la discrimination exercée, aux difficultés de réinsertion sociale; tous ces facteurs nuisent à la personne détenue.

Notre étude souligne brièvement l'importance du nombre d'individus dont la situation financière est précaire et provenant des couches socioéconomiquement défavorisées, et l'on peut se demander, devant de telles données, si l'admission en détention provisoire ne serait pas influencée partiellement par le statut de ces individus. Même si cela nous paraît inconcevable, certains éléments présentés nous laissent supposer cette assertion. 
Nous ne voudrions pas terminer cette analyse du phénomène de la détention provisoire au Québec sans reprendre à notre compte les propos de la Commission de réforme du droit du Canada (1988) qui, récemment, affirmait que les principes d'équité et de modération doivent, dans la pratique quotidienne, non seulement former l'assise des règles régissant la détention provisoire, mais aussi être mis en application. Il faut donc souhaiter le recours à une utilisation restreinte de la mesure judiciaire de la détention provisoire, au profit d'un renforcement de mesures moins ccrcitives et stigmatisantes.

\section{RÉFÉRENCES}

CAMPEAU, A., (1976), «Les prisons du Québec: ghetto de pauvres», Criminologie, vol. IX, $\mathrm{n}^{\text {os }} 1-2$, pp. $65-88$.

COMITÉ CANADIEN DE LA RÉFORME PÉNALE ET CORRECTIONNELLE (Président: R. Ouimet), (1969), Rapport du Comité canadien de la réforme pénale et correctionnelle: Justice pénale et correction : un lien à forger, Ottawa, Imprimeur de la Reine.

COMMISSION DE RÉFORME DU DROIT DU CANADA, (1988), Les mesures assurant la comparution. la mise en liberté provisoire et la détention avant le procès, document de travail 57, Ottawa, Commission de réforme du droit du Canada.

COUSINEAU, M.-M., LABERGE, D., THÉORET, B., (1986), Prisons et prisonniers : une analyse de la détention provinciale québécoise durant la dernière décennie, Québec, ministère du Solliciteur général.

DEBUYST, Ch., (1977), «Le concept de dangerosité et un de ses éléments constitutifs: la personnalité criminelle», Déviance et Société, vol. 1, n 4, pp. 363-387.

DÉVIANCE ET SOCIÉTÉ, (1986), «Débat: Entre l’ordre et les libertés: la détention provisoire», vol. $10 . \mathrm{n}^{\circ} 1$, pp. 57-63.

DOOB, A.N., CAVOUKIAN, A., (1977), "The Effect of the Revoking of Bail: R. v. Demeter», Criminal Law Quaterly, vol. 19, pp. 196-202.

DOYON, R., (1989), Étude descriptive des personnes admises au Centre de prévention de Montréal, mémoire de maîtrise, Montréal, Université de Montréal, École de criminologie.

DOZOIS, J., LALONDE, M., POUPART, J., (1984), «Dangerosité et pratique criminologique en milieu adulte», Criminologie, vol. XVII, $\mathrm{n}^{\circ} 2$, pp. 25-52.

ERICSON, R., BARANEK, (1982), The Ordering of Justice : A Study of Accused Persons as Dependants in the Criminal Process, Toronto, University of Toronto Press.

ERICSON, R., (1982), Reproducing Order: A Study of Police Patrol Work, Toronto, Ed. Butterworths.

ERICSON, R., (1981a), Making Crime: A Study of Detective Work, Toronto, Ed. Butterworths. 
ERICSON, R., (1981b), «Rules for Police Deviance», Organizational Police Deviance, Toronto, Ed. Butterworths.

FALARDEAU, J., TARASOFSKY, J., et al., (1987), Droit pénal 14, Cours de la formation professionnelle du Barreau du Québec, Cowansville, Ed. Yvon Blais.

FISCHER, J., (1986), «La pratique de la détention provisoire en République fédérale d'Allemagne», Déviance et Société, vol. 10, $\mathrm{n}^{\circ} 1$, pp. 69-74.

FRIEDLAND, M., (1965), Detention Before Trial: A Study of Criminal Cases Tried in the Toronto Magistrates' Courts, Toronto, University of Toronto Press.

GARCEAU, M.L., (1988), La détention provisoire: théorie et pratiques, mémoire de mâ̂trise, Montréal, Université du Québec à Montréal, département de sociologie.

GOLDKAMP, J., (1984), «Bail: Discrimination and Control», Criminal Justice Abstracts, March, pp. 103-127.

GOLDKAMP, J., (1979), Two Classes of Accused: A Study of Bail and Detention in American Justice, Campbridge, Mass., Ballinger Publishing Company.

GRENIER, B., TARASOFSKY, J., et al., (1987), Droit pénal 13, Cours de la formation professionnelle du Barreau du Québec, Cowansville, Ed. Yvon Blais.

HÉTU, J. (1976), «Le pauvre, la machine judiciaire et la détention», Criminologie, vol. IX, $\mathrm{n}^{\text {os }} 1-2$, pp. 87-106.

KOZA, P., DOOB, A.N., (1975:a) «The Relationship of Pre-Trial Custody to the Outcome of a Trial", Criminal Law Quaterly, vol. 17, pp. 391-400.

KOZA, P., DOOB,. A.N., (1975:b) «Some Empirical Evidence on Judicial Interim Release Proceedings", Criminal Law Quaterly, vol. 17, pp. 258-272.

LABERGE, D., LANDREVILLE, P., (1986), La détention préventive au Québec : population et procédures, document inédit présenté au Fonds pour la formation de chercheurs et l'aide à la recherche (FCAR), Montréal, Université du Québec à Montréal.

LABERGE, D., THÉORET, B., (1986), «Punir les pauvres: la culture des prisons québécoises», Sociologie du Sud-Est. Revue des Sciences Sociales, n ${ }^{\circ}$ 49-50, pp. 31-42.

LANDREVILlE, P., BLANKEVOORT, V., PIRES, A., (1981), Les coûts sociaux du système pénal, Montréal, Université de Montréal. École de criminologie.

LEIGH, L.H., (1986), «Aspects de la détention provisoire en Angleterre et au Pays de Galles», Déviance et Société, vol. 10. n 1, pp. 85-90.

LEIGHTON, B.N., DEL BUONO, V.M., (1986), «La police, les pouvoirs de la police et le maintien de l'ordre au Canada», Revue internationale de criminologie et de police technique, vol. XXXIX, $\mathrm{n}^{0} 4$, pp. 428-446.

LEVY, R., (1987), Du suspect au coupable: le travail de police judiciaire, Genève, Médecine et Hygiène.

MACKAAY, E., (1976), Les chemins de la justice: étude du fonctionnement des cours criminelles à Montréal, Montréal, Université de Montréal, Faculté de droit, Groupe de Recherche en Jurimétrie.

MARCEAU, B., (1986), «La prédiction du comportement violent : exercice nécessaire et délicat», Criminologie, vol. XIX, n², pp. 101-115.

MONTANDON, C., (1979), "La dangerosité, revue de la littérature anglo-saxonne», Déviance et Société, vol. 3, $\mathrm{n}^{0} 1$, pp. 89-104. 
MORDEN, P., (1980), «A Multivariate Analysis of the Factors Associated with Bail Decisions by Police», mémoire de maîtrise inédit, Toronto, Université de Toronto, département de criminologie.

MORSE, W., BEATTIE, R., (1932), «Survey of the Administration of Criminal Justice in Oregon», Oregon Law Review, (supp. 1932) 100, reprit ed., New-York, Arno Press, 1974.

NÉRON, J., (1988), La gestion pénale des cas de non-paiement d'amende : l' usage détourné de l'emprisonnement, mémoire de maîtrise, Montréal, Université du Québec à Montréal, département de sociologie.

POIRIER, R., (1987), «La négociation des sentences du point de vue des avocats», Criminologie, vol. $\mathrm{XX}, \mathrm{n}^{\circ} 2$, pp. 57-68.

ROBERT, Ph., (1984), La détention avant jugement: des lois et des pratiques, Paris, CESDIP.

SAINTONGE-POITEVIN, L., (1988), Code criminel et lois connexes, Montréal, Wilson et Lafleur.

SERVICES CORRECTIONNELS, (1979-80 à 1988-89), Statistiques correctionnelles du Québec, Sainte-Foy, ministère du Solliciteur général.

STATISTIQUE CANADA, (1987), Services correctionnels pour adultes au Canada, catalogue annuel 85-211, Ottawa, Centre canadien de la statistique juridique. 